Research Article

\title{
The Study of Field Equivalence Determined by the Modeled Percentage Depth Dose in Electron Beam Radiation Therapy
}

\author{
You-Guo Ma, ${ }^{1}$ Yan-Shan Zhang, ${ }^{1}$ Yan-Cheng Ye $\mathbb{D}^{,},{ }^{1}$ and Jia-Ming $\mathrm{Wu} \mathbb{D}^{1,2,3}$ \\ ${ }^{1}$ Heavy Ion Center of Wuwei Cancer Hospital, Gansu Wuwei Academy of Medical Sciences, Gansu Wuwei Tumor Hospital, \\ Wuwei City Gansu Province, China \\ ${ }^{2}$ Department of Medical Physics, Chengde Medical University, Chengde City, Hebei Province, China \\ ${ }^{3}$ Department of Radiation Oncology, Yee Zen General Hospital, Taoyuan City, Taiwan
}

Correspondence should be addressed to Yan-Cheng Ye; zlyyyyc@163.com and Jia-Ming Wu; jiaming.wu@chmsc.com

Received 4 July 2021; Revised 31 August 2021; Accepted 12 September 2021; Published 8 October 2021

Academic Editor: Maria F. Chan

Copyright (c) 2021 You-Guo Ma et al. This is an open access article distributed under the Creative Commons Attribution License, which permits unrestricted use, distribution, and reproduction in any medium, provided the original work is properly cited.

Introduction. This study presents an empirical method to model the curve of electron beam percent depth dose (PDD) by using the primary-tail function in electron beam radiation therapy. The modeling parameters $N$ and $n$ can be used to predict the minimal side length when the field size is reduced below that required for lateral scatter equilibrium (LSE) in electron radiation therapy. Methods and Materials. The electrons' PDD curves were modeled by the primary-tail function in this study. The primary function included the exponential function and the main parameters of $N$ and $\mu$, while the tail function was composed of a sigmoid function with the main parameter of $n$. The PDD of five electron energies was modeled by the primary and tail function by adjusting the parameters of $N, \mu$, and $n$. The $R_{50}$ and $R_{\mathrm{p}}$ can be derived from the modeled straight line of $80 \%$ to $20 \%$ region of PDD. The same electron energy with different cone sizes was also modeled by the primary-tail function. The stopping power of different electron energies in different depths can also be derived from the parameters $N, \mu$, and $n$. Results. The main parameters $N$ and $n$ increase but $\mu$ decreases in the primary-tail function for characterizing the electron beam PDD when the electron energy increased. The relationship of parameter $n, N$, and $\ln (-\mu)$ with electron energy are $n=31.667 E_{0}-88, N=0.9975 E_{0}-2.8535$, and $\ln (-\mu)=-0.1355 E_{0}-6.0986$, respectively. Percent depth dose was derived from the percent reading curve by multiplying the stopping power relevant to the depth in water at a certain electron energy. The stopping power of different electron energies can be derived from $n$ and $N$ with the following equation: stopping power $=\left(-0.042 \ln \left(N_{E_{0}}\right)+1.072\right) e^{\left(-\mathrm{n}_{\mathrm{E}_{0}} \cdot 5 \cdot 10^{-5}+0.0381\right) \cdot x}$, where $x$ is the depth in water. The lateral scatter equivalence (LSE) of the clinical electron beam can be described by the parameters $E_{0}, n$, and $N$ in the equation of $\mathrm{S}_{\mathrm{eq}}=$ $\left(n_{E_{0}}-N_{E_{0}}\right)^{0.288} /\left(E_{0} / n_{E_{0}}\right)^{0.0195}$. The LSE was compared with the root mean square scatter angular distribution method and shows the agreement of depth dose distributions within $\pm 2 \%$. Conclusions. The PDD of the electron beam at different energies and cone sizes can be modeled with an empirical model to deal with what is the minimal field size without changing the percent depth dose when approximate LSE is given in centimeters of water.

\section{Introduction}

The electron beam has the advantage in the target volume of superficial tumors such as skin cancers, breast cancer for chest wall irradiation, node boost dose, and head and neck cancers, while the minimizing dose to steeper tissues is widely adopted in clinical use.
The shape of the depth dose curve characteristic is the major attraction of the electron beam which offers a distinct clinical advantage over the conventional X-ray modalities for the superficial lesion. The depth dose curves, beam profiles, absolute output, and cone factor with different electron energy and cone sizes must be measured and implemented to the treatment planning system before the electron beam 
can be applied for clinical use. The electron dose distribution calculation algorithms mainly use the pencil beam model [1, 2] or another numerical model $[3,4]$ in commercial treatment planning systems [5]. The parameters of the model in pencil beams or Monte Carlo treatment planning system for electron beams need to be adjusted to fit the measurement of clinical data to perform accurate dosimetry calculation and prediction of PDD.

The pencil beams or Monte Carlo simulation algorithm describes PDD by using a complex theory with special function and $\sigma_{\mathrm{r}}(z)$ to calculate dose at any depth of $z$ [6].

Some investigators used the pencil beams or Monte Carlo simulation algorithm to investigate the changes of PDD affected by the reduction of field size smaller than the lateral scatter equilibrium (LSE) [7]. But the output factor does not change coincidently with field size increased since the PDD initially increases but finally comes to constant when the LSE is reached.

The motivation of this study we are concerned with was to reexamine the problem proposed skeptically by some investigators [8] that it was hard to find a central axis depth dose distribution just the same as any given arbitrary field in the electron beam. Fortunately, the other investigators [9] provided a solution to find the same PDD for a given electron field.

They empirically used electron energy to define the LSE, which means the depth dose distribution becomes independent of field size; therefore, we would like to visit the problem and try to find a simple way to define the minimum side length, $\mathrm{S}_{\mathrm{eq}}$, for the establishment of LSE at all depths merely by using the parameters used by the mathematic model in this study.

In this study, we proposed a simple mathematic equation to model the PDD by using the primary-tail function. Besides, we also used this empirical model to investigate the effects of field size on the central axis depth dose curve when the distance between the point of measurement and the edge of the field is shorter than the range of the laterally scattered electrons.

\section{Materials and Methods}

2.1. Electron Percent Depth Dose Numerical Equation. The primary-tail model originated from the proportion function $y(x)=1 / x$. When $x$ increases from $-\infty$ to 0 , the curve of $y$ is located in the region of the (-,-) quadrant. When $x$ goes from 0 to $+\infty$, the curve of $y$ is located in the region of the $(+,+)$ quadrant. Let $1 / x$ be $1 /|x|$, then the curve falls in the $(-,+)$ and the $(+,+)$ quadrants. The curve of $y(x)=1 /|x|$ has a left and a right tail of the dose-profile-shape pattern. Let $y(x)=1 /|x|=1 / \sqrt{\left(x^{2}\right)}$. When $x=0, y$ becomes infinite which does not happen in real dose profiles. Therefore, we insert $n$ into $y(x)$ to be $\operatorname{tail}(x)=\left(1 / \sqrt{n+\left(x^{2}\right)}\right)$, where $n>0$, let $\operatorname{tail}(x)=\left[\left(1-x / \sqrt{n^{0.5}+x^{2}}\right)+t\right]$, where $x$ is the depth in water in the unit of $\mathrm{mm}, n$ is a spreading factor of real number scalar, $t$ is a real number scalar to fine tune the height of the X-ray contamination.
On the other hand, the function $f(x)=\left(x^{0.1} /\left(N+x^{0.2}\right)\right)$ demonstrates ascending value $f$ with an increasing depth of $x$ in water. When an exponential function $e^{\mu x}$ to $f(x)$ is introduced, the combination becomes the primary function $\left(x^{0.1} /\left(N+x^{0.2}\right)\right) e^{-\mu x}$, namely, $\operatorname{primary}(x)=\left(x^{0.1} /\left(N+x^{0.2}\right)\right)$ $e^{-\mu x}$, where $x$ is the depth in water in the unit of $\mathrm{mm}$ and $N>0$ and is a harden factor of real number scalar. When $x=0, N$ plays an important role to avoid primary $(x)$ from becoming infinite, while $\mu$ is the linear attenuation factor for fine tuning the growth of the $\left(x^{0.1} /\left(N+x^{0.2}\right)\right)$ value.

Finally, the primary-tail model can be expressed as follows:

$$
\mathrm{PDD}_{p-t}=\left(\frac{x^{0.1}}{N+x^{0.2}}\right) e^{-\mu x} \cdot\left[\left(1-\frac{x}{\sqrt{n^{0.5}+x^{2}}}\right)+\mathrm{t}\right] .
$$

There are two numerical equations for describing the percent depth dose curves: the primary function and the tail function. The primary function is described as follows:

$$
\text { Primary function : }\left(\frac{x^{0.1}}{N+x^{0.2}}\right) e^{-\mu x} \text {, }
$$

where $x$ is a real number on the horizontal axis in the unit of $\mathrm{mm}$ and also denotes as the depth in water in the unit of $\mathrm{mm}, N$ is a scalar of harden factor, and $\mu$ is the linear attenuation factor in the unit of $\mathrm{mm}^{-1}$. The only scalar of the parameters $x, N$, and $\mu$ are replaced for calculation in the primary function.

$$
\text { Tail function : }\left[\left(1-\frac{x}{\sqrt{n^{0.5}+x^{2}}}\right)+t\right] \text {, }
$$

where $x$ is the same definition in equation (1) while $n$ is a spreading factor and $t$ is a factor for adjusting the height of the tail. The only scalars of the parameters $x, n$, and $t$ are replaced for calculation in the tail function.

The empirical function of percentage depth dose is the combination of these two functions, denoted as $\mathrm{PDD}_{p-t}$ :

$$
\mathrm{PDD}_{p-t}=\left(\frac{x^{0.1}}{N+x^{0.2}}\right) e^{-\mu x} \cdot\left[\left(1-\frac{x}{\sqrt{n^{0.5}+x^{2}}}\right)+t\right] .
$$

All percentage depth doses of five electron energies with different cone sizes at $\mathrm{SSD}=100 \mathrm{~cm}$ were adjusted by the main parameters of $N, n$, and $\mu$ to get the best fitting.

2.2. Experiment Design and Steps. The experiment was conducted in the following steps by using the electron beam provided by our institute's linear accelerator Varian VitalBeam (Varian, Palo Alto, CA, USA) and Elekta Infinity (Elekta, Stockholm, Sweden):

(1) The measurement of electron beam ionization depth dose curves of five energies at SSD $=100 \mathrm{~cm}$ with different cone sizes and different electron cutouts was conducted by parallel plate chamber (PTW Freiburg, 




(a)



(c)

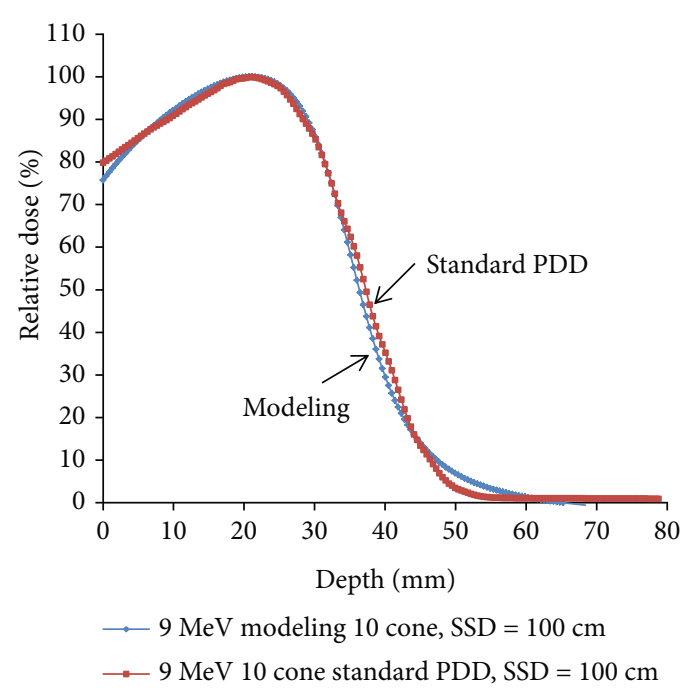

(b)

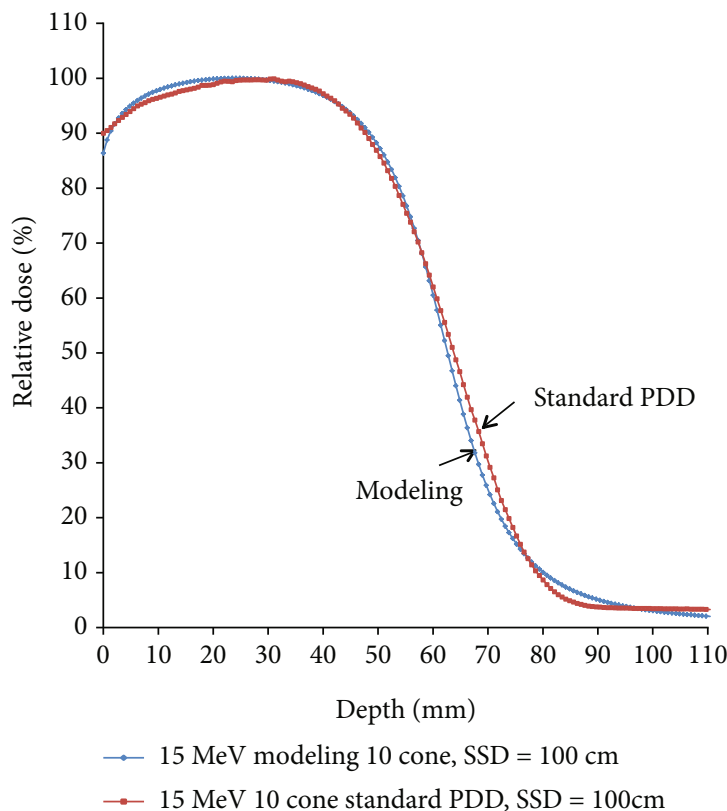

(d)

Figure 1: Continued. 


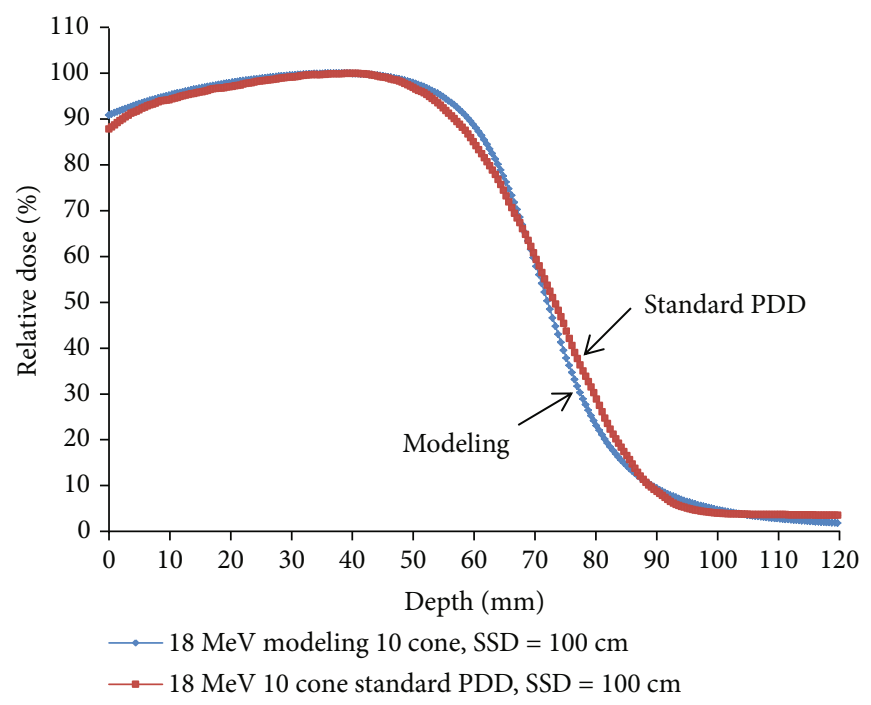

(e)

Figure 1: (a-e) Represents the fitting of the percent depth dose curve by adjusting the main parameters of $N, n$, and $\mu$ in the electron energy from 6 to $18 \mathrm{MeV}$, representatively.

Germany, TM 23343-3765) in a 3-dimensional water phantom

(2) The measurement of electron beam percent depth dose curves of five energies at $\mathrm{SSD}=100 \mathrm{~cm}$ with different cone sizes and different electrons was conducted by using the Gafchromic EBT3 films (Ashland Specialty Ingredients GP, NJ, USA; Lot \# 04022001) in a solid water phantom

(3) The empirical modeling of the electron beam percent depth dose curves was characterized by the primarytail function

(4) Logistic regression of the empirical modeling parameters of $N, \mu$, and $n$ was made for the best fitting in the primary-tail function

(5) The final step was to find a simple way to define the minimum side length, $S_{\text {eq }}$, to establish the LSE at all depths merely by using the parameters $N, n$, and $E_{0}$ used by the mathematic primary-tail model in this study

The details of each step are described in the following sections.

2.3. The Comparison of Depth Dose Curve Converted via the ionization curves measured by Farmer chamber with Gafchromic EBT3 Film. We used Gafchromic EBT3 films for the depth dose curve measurement for determining the percent depth dose measurement. The film measurements followed international protocols. A preexposure technique was used for the derivation of the calibration curve [10-12]. This was performed by giving each film a priming dose of 2 Gy to homogenize the film density using the facility of Wuwei Heavy Ion Center, Cancer Hospital (WHICH),
Gansu, China, with a dose of $1 \mathrm{~Gy}$ at the electron energy of $12 \mathrm{MeV}$. We then measured the dose homogeneity using a densitometer. Graded doses of $10 \mathrm{cGy}$ with an interval of $20 \mathrm{cGy}$ to $200 \mathrm{cGy}$ were given to the GAF chromic film to obtain the Hurter-Driffield calibration curve (H-D curve). The film was sandwiched by the solid water phantom and was irradiated with the film surface parallel to the beam central axis at SSD $100 \mathrm{~cm}$ for different cone sizes. The substance of Gafchromic provided by the vendor was assumed to be water equivalent.

All exposed films of the depth dose curve were then scanned with an Epson Expression 11000XL scanner, and the data were saved as tagged image file format (TIFF) and analyzed by the VeriSoft imaging procession software. A red filter was placed on top of the GAF films to increase the resolution of the dose-OD curves [13].

The depth dose curve derived from the ionization depth curve from the parallel-plate chamber was then compared with the depth dose curves measured by Gafchromic EBT3 films.

Absolute output and machine quality assurance were performed before conducting the measurements of percent ionization depth by parallel-plate chamber, and the percent depth dose curve was measured by the Gafchromic EBT3 film.

2.4. Ionization Depth Curve Measurement. A total of five electron energies from $6 \mathrm{MeV}$ to $18 \mathrm{MeV}$ with an increasing interval of $3 \mathrm{MeV}$ of Varian VitalBeam linear accelerator at $\mathrm{SSD}=100 \mathrm{~cm}$ and cone sizes varying from $6 \mathrm{~cm} \times 6 \mathrm{~cm}, 10$ $\mathrm{cm} \times 10 \mathrm{~cm}, \quad 15 \mathrm{~cm} \times 15 \mathrm{~cm}, 20 \mathrm{~cm} \times 20 \mathrm{~cm}$ to $25 \mathrm{~cm} \times 25$ $\mathrm{cm}$ for the measurements of depth ionization curves were carried out at WHICH in this study. Since the parallelplate chamber has a small plate separation and the charged electron particle fluence is mostly forward-directed, it is 




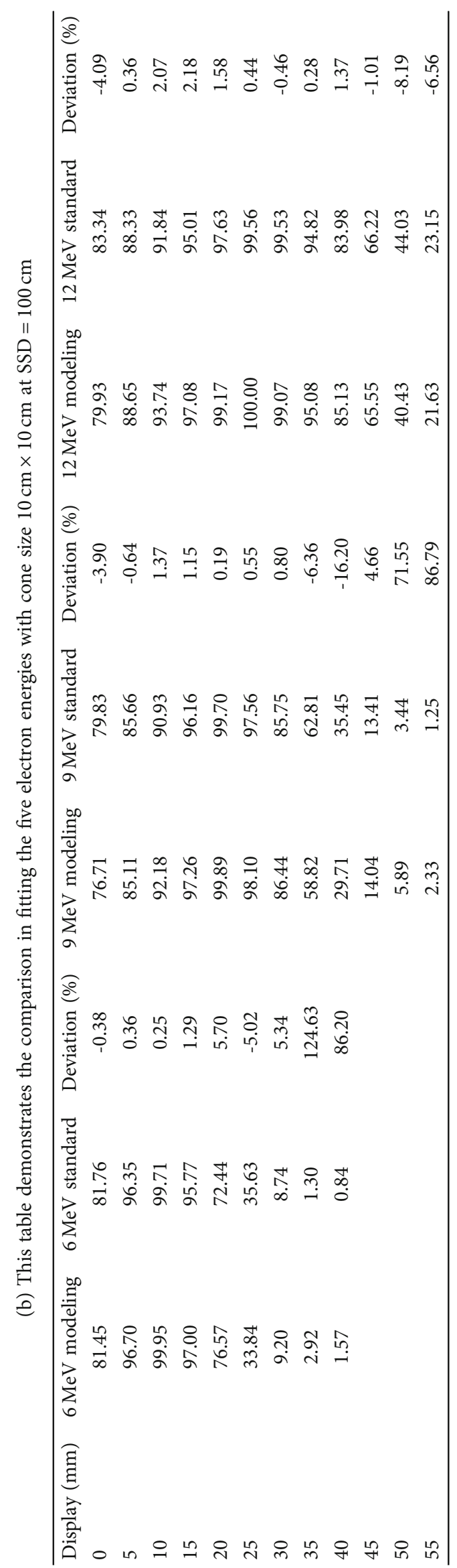




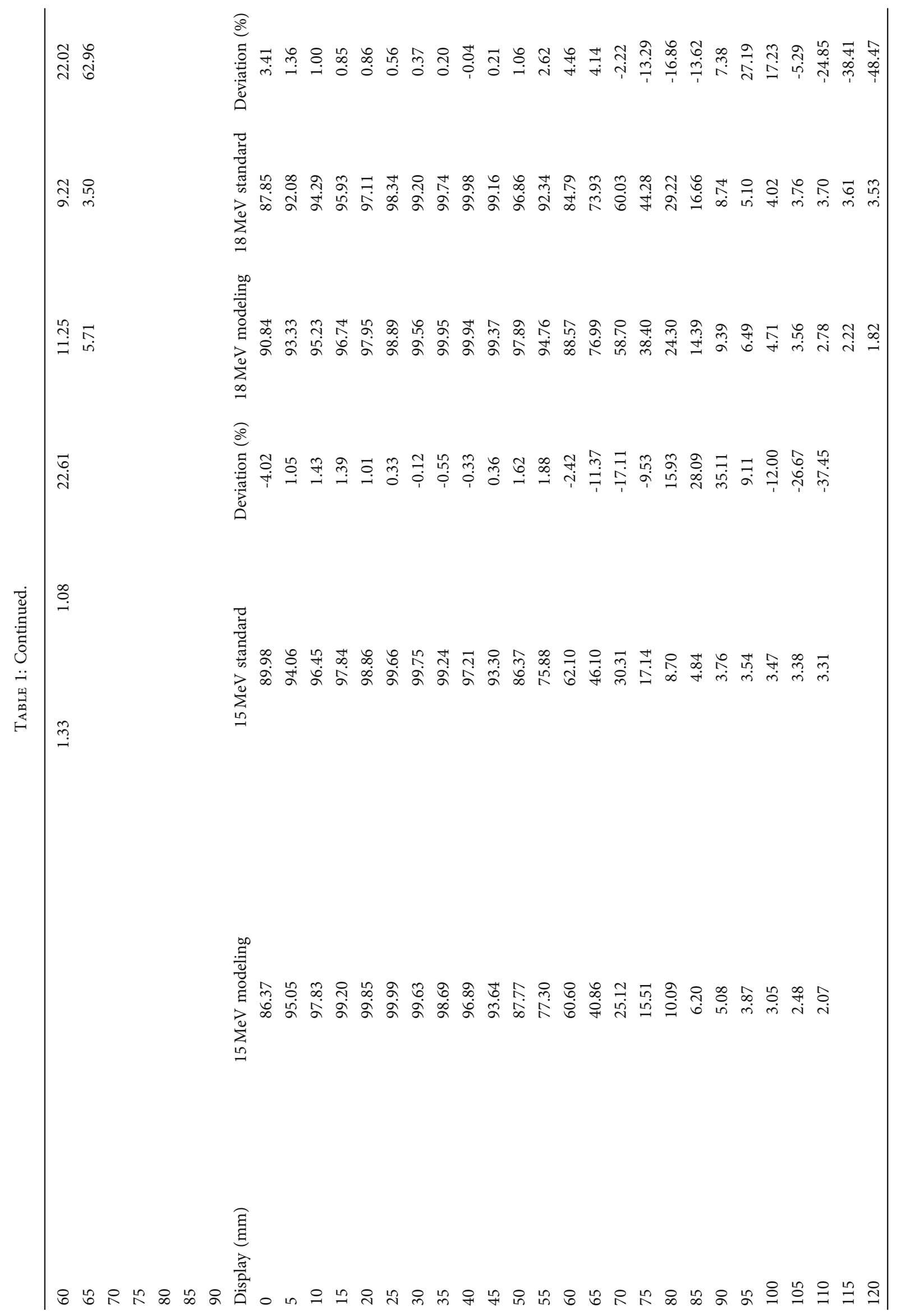


explicit that the point of measurement is the front surface of the cavity. The type of parallel-plate chamber used in this study was PTW TM 23343-3765, and the effective point of measurement was $0.3 \mathrm{~mm}$ upstream shift according to the vendor's suggestion. PTW 3D water phantom (PTW Freiburg, T41029-00006) was used for the percent ionization depth curve measurements. The depth curve measured by PTW TM 23343-3765 parallel-plate chamber was then derived to percent depth dose with the conversion function provided by the water phantom, and this percent depth dose can then be compared with the percent depth dose curve measured by the film.

For the electron, the incident of the monoenergetic spectrum is degraded as it penetrates the water, the restricted stopping power, $(L / \rho)_{\text {air }}^{\text {med }}$, increases significantly with depth. In this study, the stopping power can be derived by the $N$ and $n$ relative to its electron energy in the following equation:

Stopping power $=\left(-0.042 \ln \left(N_{E_{0}}\right)+1.072\right) e^{\left(-n_{E_{0}} \cdot 5 \cdot 10^{-5}+0.0381\right) \cdot x}$,

where $N_{E_{0}}$ and $n_{E_{0}}$ are the $N$ and $n$ at the electron energy $E_{0}$, while $x$ is the depth in water in the unit of $\mathrm{mm}$.

The relationship between $\mu$ and the slope can be calculated by the following equation:

$$
\tan ^{-1}(\text { slope })=-6.6729 \cdot \ln (\mu)-16.623 .
$$

The slope decrease when $\mu$ increases, which means the larger the electron energy, the less the side scatter is.

According to the previous investigator's study, the minimum side length for square fields, $S_{\text {eq }}$, to establish the LSE at all depths, is given by the equations of $S_{e q}$ :

$$
S_{e q}=1.58 \cdot \sqrt{E_{0}} \text {. }
$$

TABLE 2: The best fitting of the PDD modeled by the main parameters $N, n$, and $\mu$ in 5 electron energies at a cone size of $10 \mathrm{~cm} \times 10 \mathrm{~cm}$.

\begin{tabular}{lccc}
\hline Electron energy $(\mathrm{MeV})$ & $N$ & $n$ & $\mu$ \\
\hline 6 & 3.1 & 90 & 0.001 \\
9 & 6.2 & 210 & 0.0007 \\
12 & 8.9 & 290 & 0.0005 \\
15 & 12.3 & 400 & 0.0003 \\
18 & 15.1 & 470 & 0.0002 \\
\hline
\end{tabular}

Note: $N$ is a scalar of harden factor, $n$ is a scalar of the spread factor while $\mu$ is the linear attenuation factor in the unit of $\mathrm{mm}^{-1}$. The $x$ is a real number on the horizontal axis in the unit of $\mathrm{mm}, x$ also denotes the depth in water.

In our study, the minimum side length without changing the standard percent depth dose is given by the equation $\mathrm{S}_{\mathrm{eq}}$ :

$$
\mathrm{S}_{\mathrm{eq}}=\frac{\left(n_{E_{0}}-N_{E_{0}}\right)^{0.288}}{\left(E_{0} / n_{E_{0}}\right)^{0.0195}}
$$

For the specification of most probable energy, $(E)_{0}$ is defined by the Nordic Association of Clinical Physics [14] as the position of the electron fluence $\phi$ versus the energy spectral peak at the phantom surface [14] and the use of the following relationship listed:(Please romove the [14] of Physics [14] and place behind phantom surface)

$(E)_{0}=C_{1}+C_{2} R_{\mathrm{p}}+C_{3} R_{\mathrm{p}}^{2}$, where $R_{\mathrm{p}}$ is the practical range in centimeters. For water, $C_{1}=0.22 \mathrm{MeV}, \mathrm{C}_{2}=1.98 \mathrm{MeV} /$ $\mathrm{cm}$, and $\mathrm{C}_{3}=0.0025 \mathrm{MeV} / \mathrm{cm}^{2}$.

The practical range, $R_{\mathrm{p}}$, is the depth of the point where the tangent to the descending linear portion of the curve (at the point of inflection) intersects the extrapolated background.

The deviation of modeling $P D D_{P-t}$ with measured PDD at a certain depth was defined as

$$
\left(\frac{\text { the dose of modeling } \mathrm{PDD}_{P-t} \text { at a certain depth }- \text { the dose of measured PDD at the same depth }}{\text { the dose of measured PDD at the same depth }}\right) \times 100 \% \text {. }
$$

\section{Results}

A good agreement of PDD measured by film and the PDD measured by water phantom converted by the parallelplate ion chamber has been observed in this study. It is proven that the energy independence of the film which may be due to the collision stopping power in emulsion and in water varies slowly with the electron energy.

\subsection{The Best Fitting of Percent Depth Dose by Empirical} Function in Five Electron Energies. The percent depth dose of the electron beam with different energies adopted in this study was already measured by the water phantom at the commission of the linear accelerator. By adjusting the main parameters of $N, n$, and $\mu$, we get the best fitting of allelectron percent depth dose curves of every energy with cone size $10 \mathrm{~cm} \times 10 \mathrm{~cm}$ in Figure 1 .

3.2. The Percent Depth Dose Was Fitted by Empirical Function in the Same Electron Energy with Different Cone Sizes. The percent depth dose varies slightly in the same electron energy with different cone sizes; therefore, we tabulated the deviation of measured and modeled with one of the electron energy of $12 \mathrm{MeV}$.

Table 1(a) demonstrates the comparison in fitting the same electron energy of $12 \mathrm{MeV}$ in 5 different cone sizes from $6 \mathrm{~cm} \times 6 \mathrm{~cm}$ to $25 \mathrm{~cm} \times 25 \mathrm{~cm}$ at $\mathrm{SSD}=100 \mathrm{~cm}$. 


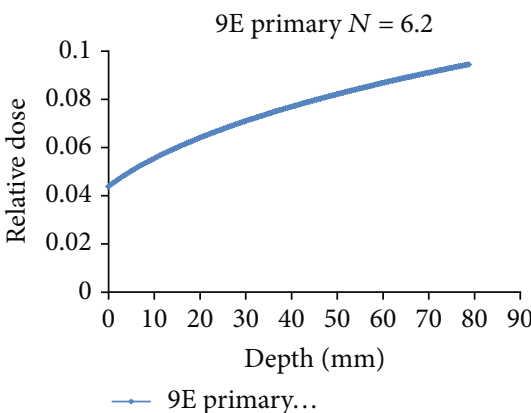

(a)

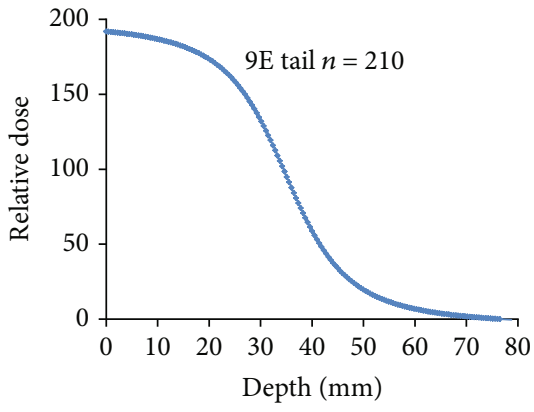

(b)



(c)

FIgURE 2: Demonstrates how the primary function is affected by parameters $N$ and $n$; the best fitting of parameter $N$ and $n$ in the PDD of $9 \mathrm{MeV}$ electron beam in (a) and (b). The curve becomes less attenuated in a large $N$ as shown at the bottom in (c). The factor $n$ represents the spreading factor at any electron energy; it is explicitly a large electron beam that comes with a large $n$ as shown in Table 2 and (c). The factor $\mu$ is used to fine-tune the shape of the PDD curve, and it is trending to be smaller in a high electron energy beam.

The comparison error between the modeling and the measurements for different electron energies but the same electron size of $10 \mathrm{~cm} \times 10 \mathrm{~cm}$ is listed in Table $1(\mathrm{~b})$.

3.3. The Best Fitting of Percent Depth Dose by the Main Parameters of $N, n$, and $\mu$ in the Empirical Function of AllElectron Energy with Different Cone Sizes. Table 2 shows the main parameters of $N, n$, and $\mu$ of the best fitting of all-electron percent depth dose curves at every energy with different cone sizes. According to Table 2, $N$ and $n$ increases while $\mu$ decreases when electron energy increases.

By using the primary-tail function for modeling electron energy at $12 \mathrm{MeV}$ in different cone sizes, the measured PDD can be characterized perfectly than the other electron energies. For the overall fitting of the measured PDD, we found that the intersection of the X-ray contamination with the
PDD descending portion at the turning point was pretty bad which was the limitation of the primary-tail function. It might be caused by a lack of scattering algorithm consideration at the junction of the X-ray contamination and the end of the PDD curve in the current $\mathrm{PDD}_{P-t}$ model. The solution to this problem will be the next study topic in our future investigation.

\section{Discussion}

The percent depth dose can be fitted quite well with the primary-tail modeling by adjusting the main parameters of $N, n$, and $\mu$ in five electron energies except for the descending curve intersection area with $\mathrm{X}$-ray contamination in Figure 1. 
TABLE 3: The maximum ionizing depth, dose maximum depth, and mean energy in five different electron energies with $10 \mathrm{~cm} \times 10 \mathrm{~cm}, E_{0}$ $\left(E_{0}=2.33 \mathrm{MeV} / \mathrm{cm} \cdot R_{50}\right)$ is shown. The depth at which the dose is $50 \%$ of the maximum dose is defined as $R_{50}$ in this study.

\begin{tabular}{lcccccc}
\hline Nominal energy $(\mathrm{MeV})$ & $E_{0}(\mathrm{MeV})$ & $R_{\max } / d_{\max }(\mathrm{mm})$ & $R_{90}(\mathrm{~mm})$ & $R_{50}(\mathrm{~mm})$ & $R_{\mathrm{p}}(\mathrm{mm})$ & X-ray contamination $(\%)$ \\
\hline 6 & 5.41 & $11.1 / 12.1$ & 16.9 & 23.2 & 30.2 & 0.7 \\
9 & 8.66 & $20.6 / 21.5$ & 28.3 & 37.1 & 45.1 & 1.2 \\
12 & 11.27 & $26.1 / 27.1$ & 37.4 & 48.3 & 58.7 & 2.1 \\
15 & 14.04 & $27.1 / 28.4$ & 46.1 & 60.3 & 73.7 & 2.6 \\
18 & 17.03 & $24.3 / 25.2$ & 56.8 & 73.1 & 88.7 & 3.5 \\
\hline
\end{tabular}

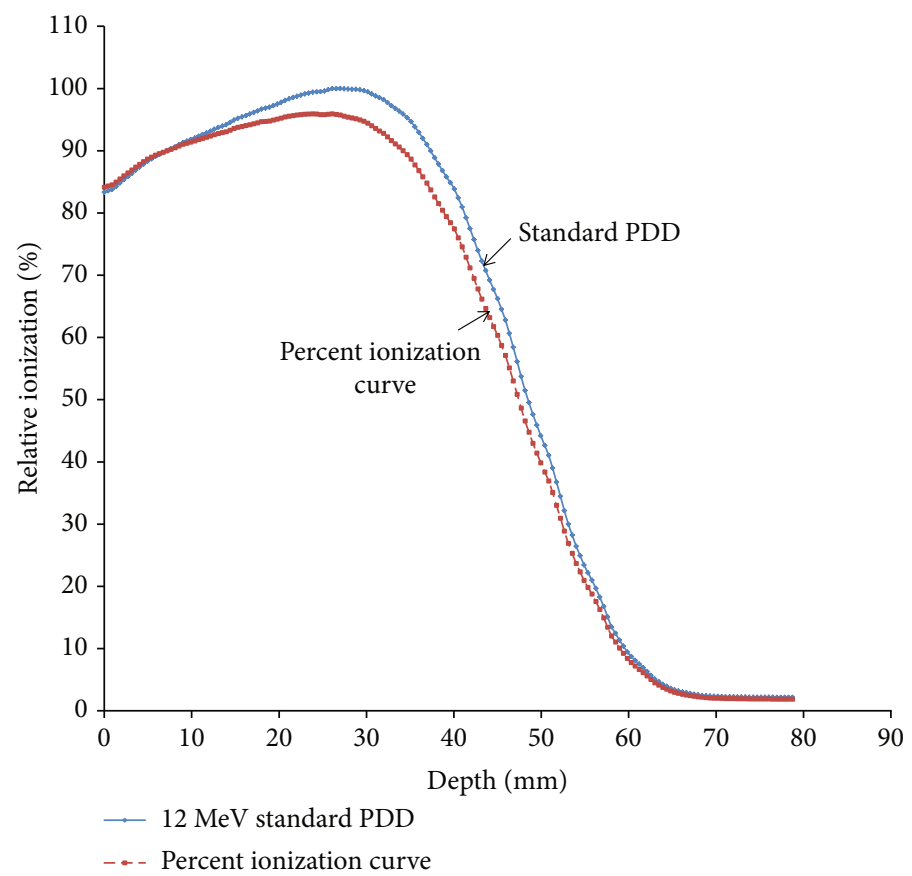

FIgURE 3: The converted ionization depth curve was calculated by dividing the water phantom of PDD at 10 cones with the relative stopping power from the above calculation at any certain depth for $12 \mathrm{MeV}$.

Tables 1(a) and 1(b) demonstrate the comparison in fitting the electron energy of $12 \mathrm{MeV}$ with 5 cone sizes from $6 \mathrm{~cm} \times 6 \mathrm{~cm}$ to $25 \mathrm{~cm} \times 25 \mathrm{~cm}$ at $\mathrm{SSD}=100 \mathrm{~cm}$ while the ranges of error between the modeling and the measurements for different electron energies at the same cone size of 10 $\mathrm{cm} \times 10 \mathrm{~cm}$ are listed in Table 1(b). A significant error between the model and the measurements was found at the turning point of the PDD curve's descending portion intersecting with the X-ray contamination while this is irrelevant when examined the $R_{\mathrm{p}}$, but it is a limitation of the primary-tail model indeed.

The impact in clinical of the large deviation at the turning point of the PDD descending potion to X-ray contamination intersection is insignificant when examining $R_{\mathrm{p}}$. Secondly, the purpose of this study is to investigate the field equivalence in the electron beam; the disagreement at the turning point is located at the end of the central axis depth dose curve; therefore, the influence of the large deviation to the point of measurement and the shorter edge than the range of the laterally scattered is irrelevant. Finally, the mon- itor unit calculation is indifferent to the presence of the large deviation at the turning point since $90 \%$ isodose curve is usually selected for MU calculation for dose delivery in electron beam therapy.

The more electron energy, the more $N, n$, and $\mu$ it has for the best fitting of the PDD curve as shown in Table 2 .

Figures 2(a) and 2(b) show the best fitting of parameters $N$ and $n$ in the PDD of $9 \mathrm{MeV}$ electron beam, respectively. We can adjust the value of $N$ to fit the measured PDD. Since $\mathrm{N}$ is the hardened factor, more electron energy comes with a large value of $N$ as shown in Table 2, and the curve becomes less attenuated in a large $N$ as shown at the bottom in Figure 2(c). The factor $n$ represents the spreading factor at any electron energy; it is explicitly a large electron beam that comes with a large $n$ as shown in Table 2 and Figure 2(c). The factor $\mu$ is used for fine-tuning the shape of the PDD curve, and it is trending to be smaller in a high electron energy beam as shown in Table 2 .

In this study, we defined the practical range, $R_{\mathrm{p}}$, as the depth of the point where the tangent to the descending 


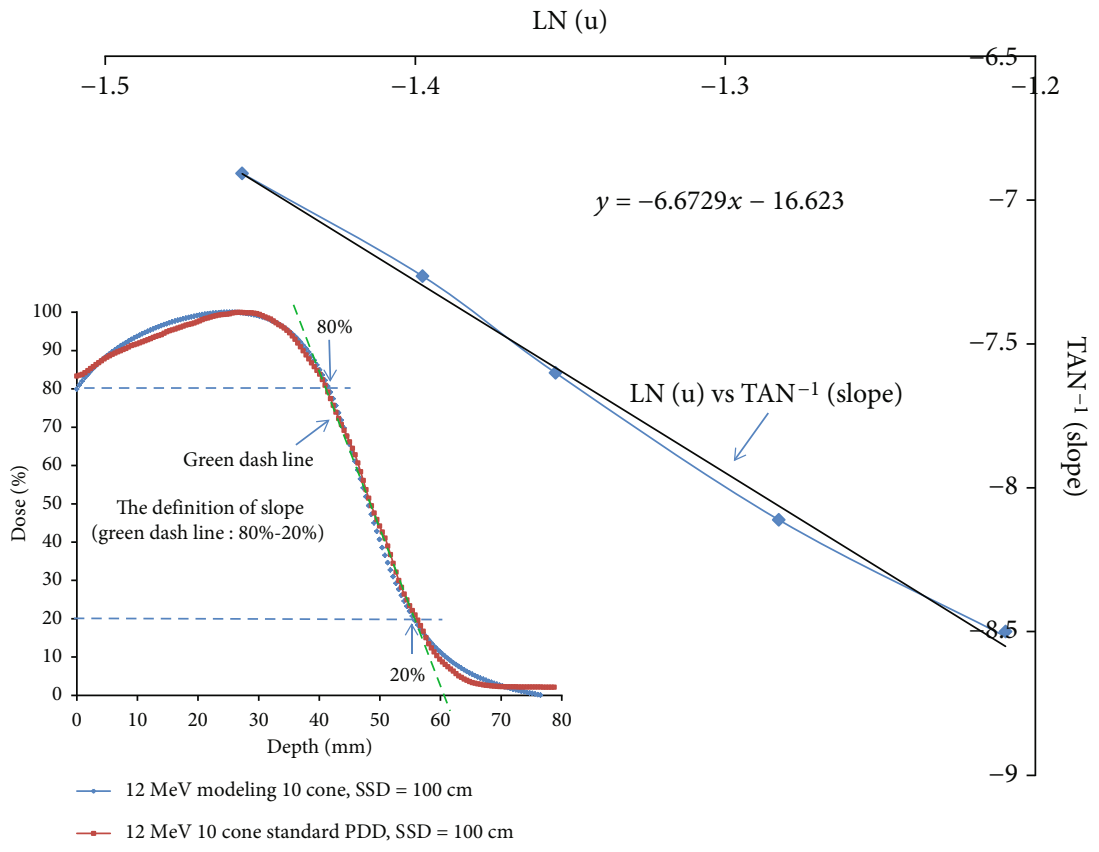

FIGURE 4: The relationship of the slope of descending portion ranged in between $80 \%$ and $20 \%$ of the electron PDD at cone size $10 \mathrm{~cm} \times 10 \mathrm{~cm}$ is relative to the electron energy. The relationship between $\mu$ and slope can be calculated by the equation, $\tan ^{-1}(\mathrm{slope})=$ $-6.6729 \cdot \ln (-\mu)-16.623$; as the figure shows, the slope decreases when the $\mu$ increases, which means the larger the electron energy, the less the side scatter it is.

linear portion of $80 \%$ to $20 \%$ of the curve intersects the extrapolated X-ray contamination at the point of inflection. The depth at which the dose is $50 \%$ of the maximum dose is defined as $R_{50}$, and the mean energy of the electron beam, $E_{0}$, is equaled to $R_{50} \times 2.33 \mathrm{MeV} / \mathrm{cm}$. Table 3 shows the maximum reading depth, dose maximum depth, and mean energy of the electron beam, $E_{0}\left(E_{0}=2.33 \mathrm{MeV} / \mathrm{cm} \cdot R_{50}\right)$. The depth at which the dose is $50 \%$ and $90 \%$ of the maximum dose is defined as $R_{50}$ and $R_{90}$, respectively, as the American Association of Physicists in Medicine (AAPM) protocol recommended [15] in this study.

The depth dose curve converted by ionization depth curve was calculated by dividing the standard PDD measured by water phantom with the relative stopping power at a certain depth for $12 \mathrm{MeV}$ and is shown in Figure 3.

The angle of the descending portion ranged in between $80 \%$ and $20 \%$ of cone size $10 \mathrm{~cm} \times 10 \mathrm{~cm}$ at any electron energy is defined as the slope of the electron PDD.

The relationship between $\mu$ and slope is shown in Figure 4 and can be calculated by equation ((5). The modeled percent depth dose curve as well as the standard percent depth dose curves varied by a little deviation with the field size at the same electron energy of $12 \mathrm{MeV}$ in Table 1 (a).

In electron beam therapy, if the X-ray jaw setting were changed with the treatment field, the percent depth dose curve would vary a wide range with field size, especially for lower energy beams. In clinical practice, electron therapy usually provides a fixed jaw opening, and the treatment field size is varied by various cone sizes. Such an arrangement minimizes the variation of collimator scatter, and therefore, the PDD variation with field size is kept reasonably small if the field size is not reduced below that required for lateral scatter equilibrium (LSE).

The effects of field size on output and the change of PDD due to phantom scatter is significant when the field is shorter than the range of the laterally scattered electrons. After this distance is reached, there is no further increase in depth dose caused by phantom scatter. When the field size of electron cutout is reduced below that required for LSE, the change of PDD is obvious and the dose rate decreases rapidly as well. The change of PDD is shown in Figure 4. In these measurements, the field size at the phantom surface was fabricated by electron cutout, which, therefore, varied without changing the photon jaw setting. For small fields, the shape of the depth dose, as well as output factor, can be significantly reduced compared with the broad beam distribution.

Figure 4 shows that the relationship between $\mu$ and the slope can be calculated by equation (5); the slope decreases when the $\mu$ increases, which means the larger the electron energy, the less the side scatter is.

When the field size is reduced by the electron cutout but keeps a fixed jaw opening, the depth dose curves move toward the left in Figure 5, and the $d_{\max }$ shifts toward the surface for the smaller fields less than $4 \mathrm{~cm}^{2}$.

As the field size is increased in electron beam radiotherapy, the PDD initially increases but becomes constant beyond a certain field size.

Table 4 makes the comparison of $\mathrm{S}_{\mathrm{eq}}$ and $S_{e q}$. According to the calculation from $S_{\text {eq }}$, the minimum side lengths without changing the standard percent depth dose of $9 \mathrm{MeV}$ and $15 \mathrm{MeV}$ are $4.9 \mathrm{~cm}$ and $5.9 \mathrm{~cm}$, respectively. 


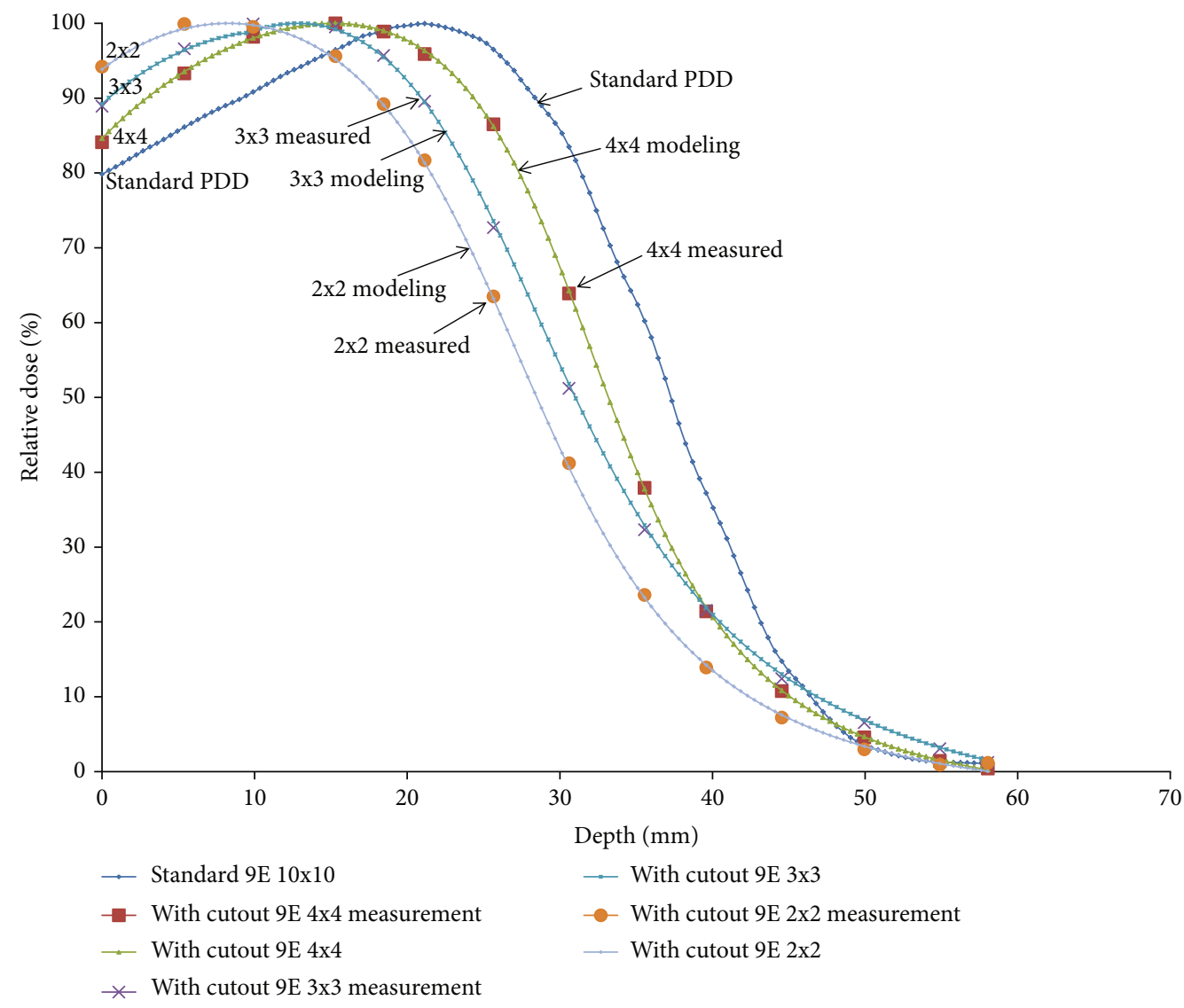

Figure 5: This figure shows the depth dose curves move toward the left significantly when the field size is reduced to less than $4.9 \mathrm{~cm}$ in this figure. Thus, the depth dose distribution for small fields is field size-dependent, while for the field that was required for lateral scatter equilibrium (LSE), it is independent of field size.

TABLE 4: The comparison ofS ${ }_{\text {eq }}$ and $S_{e q}$, the minimum side lengths to remain the standard percent depth doses of $9 \mathrm{MeV}$ and $15 \mathrm{MeV}$ unchanged are $4.9 \mathrm{~cm}$ and $5.9 \mathrm{~cm}$, respectively.

\begin{tabular}{lccccccccc}
\hline $\begin{array}{l}\text { Energy }(\mathrm{MeV}) \\
\text { (nominal) }\end{array}$ & $E_{0}(\mathrm{MeV})$ & $n$ & $N$ & Slope $(80 \%-20 \%)$ & $\tan ^{-1}($ slope $)$ & $\mu$ & $\ln (\mu)$ & $\begin{array}{c}\mathrm{S}_{\text {eq }}(\mathrm{mm}, \text { this } \\
\text { study) }\end{array}$ & $\begin{array}{c}S_{\text {eq }}=1.58 \sqrt{E_{0}} \\
(\mathrm{~mm})\end{array}$ \\
\hline 6 & 5.41 & 90 & 3.1 & -8.6593 & -1.45582 & 0.001 & -6.9077 & 38.13 & 36.75 \\
9 & 8.66 & 210 & 6.2 & -5.7176 & -1.39765 & 0.0007 & -7.2644 & 49.17 & 46.50 \\
12 & 11.27 & 290 & 8.9 & -4.5559 & -1.35473 & 0.0005 & -7.6009 & 53.98 & 53.04 \\
15 & 14.04 & 400 & 12.3 & -3.3749 & -1.28273 & 0.0003 & -8.1117 & 59.33 & 59.20 \\
18 & 17.03 & 470 & 15.1 & -2.6474 & -1.20964 & 0.0002 & -8.5171 & 62.10 & 65.20 \\
\hline
\end{tabular}

The depth dose curves move toward the left significantly when the field size is reduced to less than $4.9 \mathrm{~cm}$ and $5.9 \mathrm{~cm}$ of $9 \mathrm{MeV}$ in Figure 5 and $15 \mathrm{MeV}$ in Figure 6, respectively. Thus, the depth dose distribution for small fields is field size-dependent, while for a field that is required for lateral scatter equilibrium (LSE), it is independent of field size.

The essential valuable contribution of this study is the modeling techniques and the establishment of lateral scatter equivalence (LSE) in the clinic using electron beams. The lateral scatter equilibrium for the electron field can be calculated and achieved by using the simple mathematic function presented in this study.
One of the great successes in this study is to provide a home-generated calculation method to determine the equivalent side of a square field that satisfies the establishment of lateral scatter equilibrium so that it keeps the same depth dose distribution for electron cutout fields in clinical use.

The method described in this study provides only for the determination of the equivalent side of a square field but does not include the correction of output factors; therefore, more parameters such as incident fluence, size of the applicator, and electron energy need to be measured to do the correction of output factor [16]. 


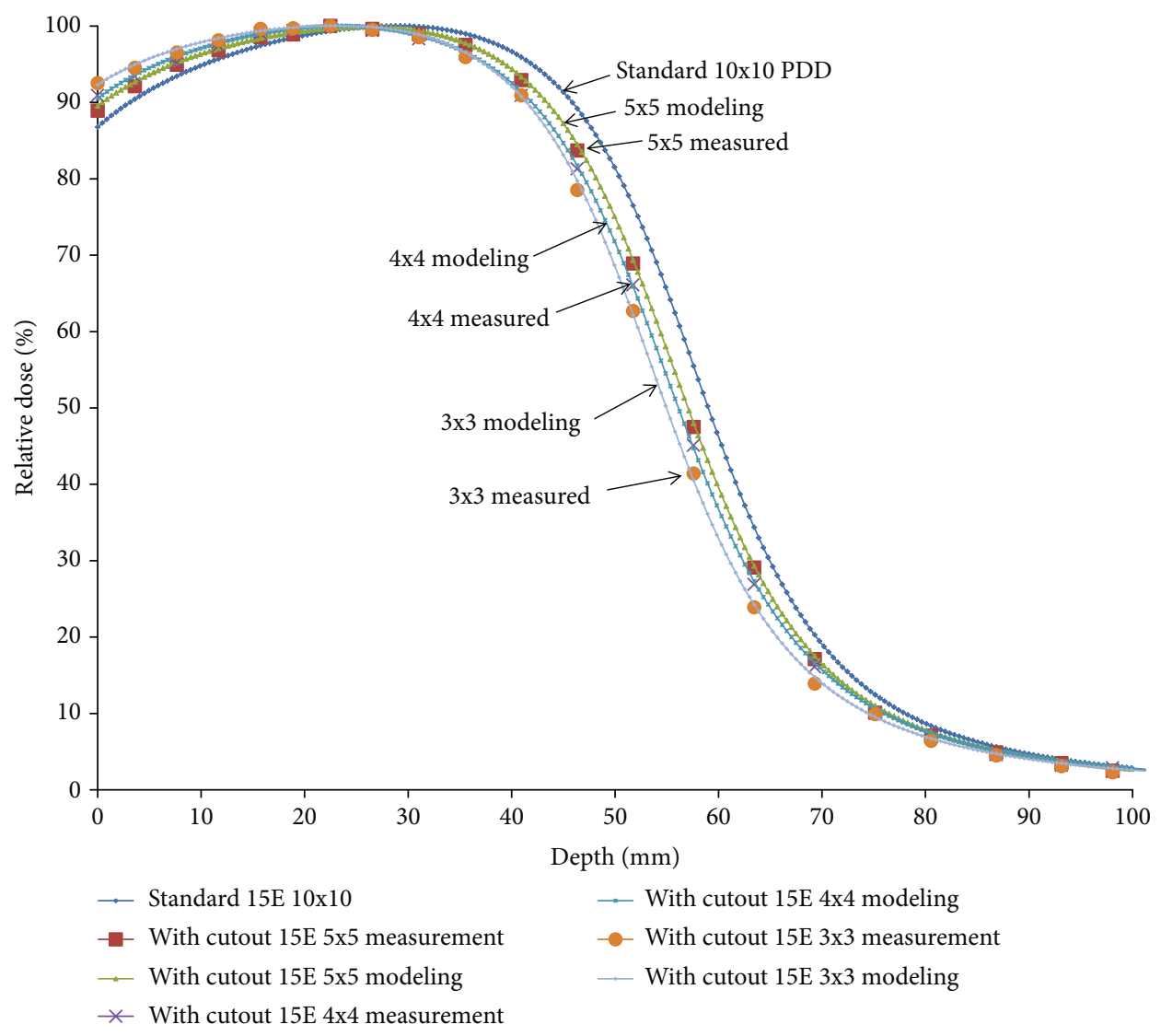

Figure 6: The depth dose curves move toward the left significantly when the field size is reduced to less than $5.9 \mathrm{~cm}$ of $15 \mathrm{MeV}$. It is concluded that for the field that is required for lateral scatter equilibrium (LSE) to remain PDD constant, once the LSE is reached, PDD is independent of field size.

\section{Conclusions}

In this study, we presented a study with an empirical method to model the electron beam percent depth dose (PDD) curve by using the primary function and tail function in radiation therapy. The modeling parameters $N$ and $n$ can be used to predict the minimal side length when the field size is reduced below that required for lateral scatter equilibrium (LSE) in electron radiation therapy.

Useful criteria have been developed to predict a lack of achievement of lateral scatter equilibrium to check for a field if whether the patient treatment monitor unit needs to be modified or not.

\section{Data Availability}

The data used to support the findings of this study are available from the corresponding authors upon request.

\section{Conflicts of Interest}

There are no actual or potential conflicts of interest in this study.

\section{Authors' Contributions}

You-Guo Ma and Yan-Shan Zhang contributed equally to this work.

\section{Acknowledgments}

The authors appreciate Professor Zhang Yan Shan and Professor Ye Yan Cheng for their great contribution to this study. Professor Ye Yan Cheng is juxtaposed with the correspondence author (co-corresponding authors with equal contribution). This work was supported by the following funding: Key R\&D Plan of the Science and Technology Program of Gansu Province, China (18YF1FH153).

\section{References}

[1] K. R. Hogstrom, M. D. Mills, and P. R. Almond, "Electron beam dose calculations," Physics in Medicine \& Biology, vol. 26, no. 3, pp. 445-459, 1981.

[2] K. R. Hogstrom, M. D. Mills, J. A. Eyer et al., "Dosimetric evaluation of a pencil-beam algorithm for electrons employing a two-dimensional heterogeneity correction," International Journal of Radiation Oncology • Biology • Physics, vol. 10, no. 4, pp. 561-569, 1984. 
[3] J. E. Cygler, G. M. Daskalov, and G. H. Chan, "Evaluation of the first commercial Monte Carlo dose calculation engine for electron beam treatment planning," Medical Physics, vol. 31, no. 1, pp. 142-153, 2004.

[4] P. Pemler, J. Besserer, U. Schneider, and H. Neuenschwander, "Evaluation eines kommerziellen Monte-Carlo-Bestrahlungsplanungssystems fur Elektronen (eMC)," Zeitschrift für Medizinische Physik, vol. 16, no. 4, pp. 313-329, 2006.

[5] G. X. Ding, J. E. Cygler, C. W. Yu, N. I. Kalach, and G. Daskalov, "A comparison of electron beam dose calculation accuracy between treatment planning systems using either a pencil beam or a Monte Carlo algorithm," International Journal of Radiation Oncology • Biology • Physics, vol. 63, no. 2, pp. 622-633, 2005.

[6] H. Neuenschwander, T. R. Mackie, and P. J. Reckwerdt, "MMC-a high-performance Monte Carlo code for electron beam treatment planning," Physics in Medicine and Biology, vol. 40, no. 4, pp. 543-574, 1995.

[7] F. M. Khan and P. D. Higgins, "Calculation of depth dose and dose per monitor unit for irregularly shaped electron fields: an addendum," Physics in Medicine and Biology, vol. 44, no. 6, pp. N77-N80, 1999.

[8] K. R. Hogstrom, R. G. Kurup, A. S. Shiu, and G. Starkschall, “A two-dimensional pencil-beam algorithm for calculation of arc electron dose distributions," Physics in Medicine and Biology, vol. 34, no. 3, pp. 315-341, 1989.

[9] F. M. Khan and P. D. Higgins, "Field equivalence for clinical electron beams," Physics in Medicine and Biology, vol. 46, no. 1, pp. 9-14, 2001.

[10] R. Dreindl, D. Georg, and M. Stock, "Dosimetrie mit radiochromatischen Filmen: Betrachtungen zur prazisen und genauen dosimetrischen Verifikation mit EBT2- und EBT3Filmen," Zeitschrift für Medizinische Physik, vol. 24, no. 2, pp. 153-163, 2014.

[11] A. Niroomand-Rad, S. T. Chiu-Tsao, M. P. Grams et al., "Report of AAPM Task Group 235 Radiochromic film dosimetry: an update to TG-55," Medical Physics, vol. 47, no. 12, pp. 5986-6025, 2020.

[12] D. Lewis, A. Micke, X. Yu, and M. F. Chan, "An efficient protocol for radiochromic film dosimetry combining calibration and measurement in a single scan," Medical Physics, vol. 39, no. 10, pp. 6339-6350, 2012.

[13] O. A. García-Garduño, J. M. Lárraga-Gutiérrez, M. RodríguezVillafuerte, A. Martínez-Dávalos, and T. Rivera-Montalvo, "Effect of correction methods of radiochromic EBT2 films on the accuracy of IMRT QA," Applied Radiation and Isotopes, vol. 107, pp. 121-126, 2016.

[14] Nordic Association of Clinical Physics, "Procedures in external radiation therapy dosimetry with electron and photon beams with maximum energies between 1 and $50 \mathrm{MeV}$," Acta Radiologica, vol. 19, p. 55, 1980.

[15] B. J. Gerbi, J. A. Antolak, F. C. Deibel et al., "Recommendations for clinical electron beam dosimetry: supplement to the recommendations of Task Group 25," Medical physics, vol. 36, no. 7, pp. 3239-3279, 2009.

[16] F. M. Khan, P. D. Higgins, B. J. Gerbi, F. C. Deibel, A. Sethi, and D. N. Mihailidis, "Calculation of depth dose and dose per monitor unit for irregularly shaped electron fields," Medical Biology, vol. 43, no. 10, pp. 2741-2754, 1998. 